\title{
Semicircles in the Arbelos with Overhang and Division by Zero
}

\section{Semicircles in the Arbelos with Overhang and Division by Zero}

\section{ABSTRACT}

We consider special semicircles, whose endpoints lie on a circle, for a generalized arbelos called the arbelos with overhang considered in [4] with division by zero.

Key words: arbelos, arbelos with overhang, Aida arbelos, semicircle touching at the endpoints, insemicircle, Archimedean semicircle, division by zero

MSC2010: 01A27 51M04

\section{Polukružnice $u$ arbelosima s produžecima i} dijeljenje s nulom

\section{SAŽETAK}

U radu proučavamo posebne polukružnice, one čije krajnje točke leže na jednoj kružnici, u poopćenim arbelosima $s$ produžecima kao u [4] uz korištenje dijeljenja s nulom.

Ključne riječi: arbelosi, arbelosi s produžecima, Aida arbelosi, polukružnice s diranjem u krajnjim točkama, unutarnje polukružnice, Arhimedove polukružnice, dijeljenje s nulom

\section{Introduction}

For a point $O$ on the segment $A B$ such that $|A O|=2 a$, $|B O|=2 b$, let $A_{h}$ (resp. $B_{h}$ ) be a point on the half line $O A$ (resp. $O B$ ) with initial point $O$ such that $\left|O A_{h}\right|=2(a+h)$ (resp. $\left|O B_{h}\right|=2(b+h)$ ) for a real number $h$ satisfying $-\min (a, b)<h$. In [4] we have considered a generalized arbelos consisting of the three semicircles $\alpha, \beta$ and $\gamma$ of diameters $A_{h} O, B_{h} O$ and $A B$, respectively, constructed on the same side of $A B$. The figure is denoted by $(\alpha, \beta, \gamma)_{h}$ and is called the arbelos with overhang $h$ (see Figure 1). The ordinary arbelos is obtained from $(\alpha, \beta, \gamma)_{h}$ if $h=0$, which is denoted by $(\alpha, \beta, \gamma)_{0}$.

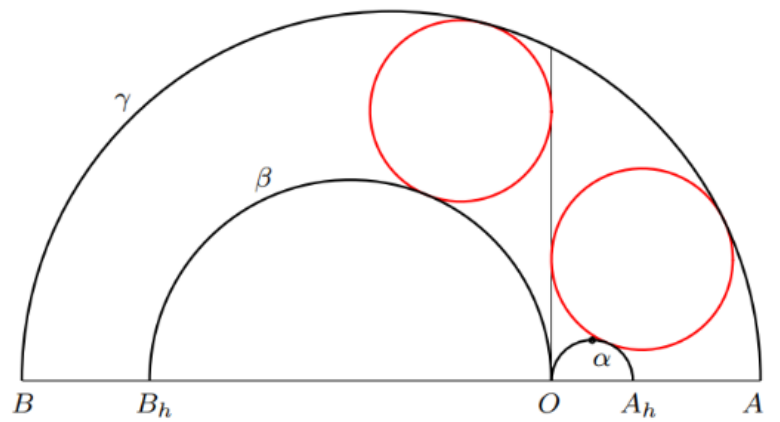

Figure 1: $(\alpha, \beta, \gamma)_{h},-\min (a, b)<h<0$.

Let $c=a+b$. The circle touching $\alpha$ (resp. $\beta$ ) externally, $\gamma$ internally, and the axis from the side opposite to $B$ (resp. $A$ ) has radius

$$
r_{\mathrm{A}}=\frac{a b}{c+h} .
$$

The two circles are called the twin circles of Archimedes of $(\alpha, \beta, \gamma)_{h}$. Circles of radius $r_{\mathrm{A}}$ are called Archimedean circles of $(\alpha, \beta, \gamma)_{h}$ or said to be Archimedean with respect to $(\alpha, \beta, \gamma)_{h}$.

In this article we consider special semicircles, which are counterpart to the incircle and Archimedean circles of $(\alpha, \beta, \gamma)_{h}$ using division by zero. At the last part of this paper we consider special case of $(\alpha, \beta, \gamma)_{h}$ considered by Aida [1]. We consider using a rectangular coordinate system with origin $O$ such that the farthest point on $\alpha$ have coordinates $(a+h, a+h)$ (see Figure 1). The radical axis of $\alpha$ and $\beta$ is called the axis.

\section{Incircle and insemicircle}

In this section we consider the incircle of $(\alpha, \beta, \gamma)_{h}$ and an inscribed semicircle in $(\alpha, \beta, \gamma)_{h}$. If a circle touches $\alpha$ and 
$\beta$ externally and $\gamma$ internally, we call the circle the incircle of $(\alpha, \beta, \gamma)_{h}$ (see Figure 2). If the endpoints of a semicircles lie on a circle, we say that the semicircle touches the circle at the endpoints. If a semicircle touches $\alpha$ and $\beta$, and $\gamma$ at the endpoints, we say that the semicircle is inscribed in $(\alpha, \beta, \gamma)_{h}$. We have considered such a semicircle in [2] for $(\alpha, \beta, \gamma)_{0}$. We use the next proposition.

Proposition 1 A semicircle of radius s touches a circle of radius $r$ at the endpoints if and only if $d^{2}+s^{2}=r^{2}$, where $d$ is the distance between the centers of the semicircle and the circle.

Let $v=\sqrt{(c+h)^{2}-2 a b+h^{2}}$.

Theorem 1 The following statements hold.

(i) The incircle of $(\alpha, \beta, \gamma)_{h}$ has radius

$i_{c}=\frac{a b(c+2 h)}{(c+h)^{2}-a b}$.

(ii) If a semicircle is inscribed in $(\alpha, \beta, \gamma)_{h}$, then it has radius

$i_{s}=\frac{-v^{2}+\sqrt{8 a b(c+2 h)^{2}+v^{4}}}{2(c+2 h)}$.

Proof. We prove (ii). Let $(x, y)$ and $i_{s}$ be the coordinates of the center and the radius of the semicircle inscribed in $(\alpha, \beta, \gamma)_{h}$. Then we get $(x-(a+h))^{2}+y^{2}=$ $\left((a+h)+i_{s}\right)^{2},(x+(b+h))^{2}+y^{2}=\left((b+h)+i_{s}\right)^{2}$ and $(x-(a-b))^{2}+y^{2}+i_{s}^{2}=c^{2}$ by Proposition 1 Eliminating $x$ and $y$ from the three equations and solving the resulting equation for $i_{s}$, we get (2). The part (i) is proved similarly.

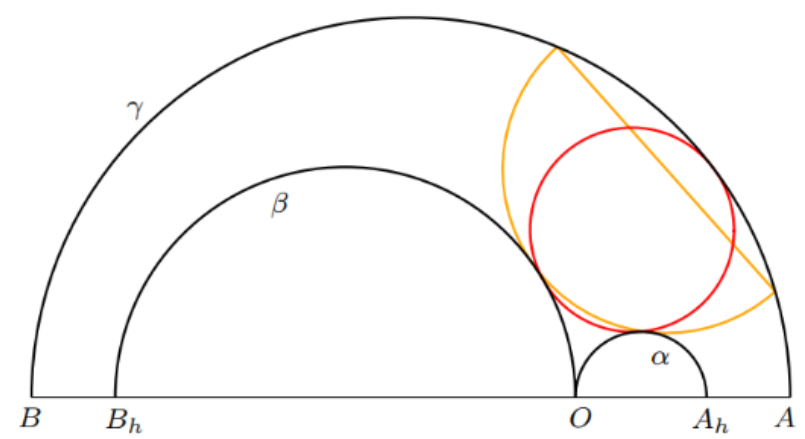

Figure 2

The theorem shows that an inscribed semicircle in $(\alpha, \beta, \gamma)_{h}$ is determined uniquely. Hence we can call it the insemicircle of $(\alpha, \beta, \gamma)_{h}$.

We consider a condition where a semicircle of radius $i_{s}$ touches $\gamma$. If one of the endpoints of a semicircle $S_{1}$ lies on a semicircle $S_{2}$ and the other endpoints of $S_{1}$ lies on the reflection of $S_{2}$ in its diameter, we still say that $S_{1}$ touches $S_{2}$ at the endpoints. The circle of center of coordinates $((a+h) m, 0)$ (resp. $(-(b+h) n, 0)$ and passing through $O$ is denoted by $\alpha_{m}$ (resp. $\beta_{n}$ ) for a real number $m$ (resp. $\left.n\right)$ (see Figure 3). For points $P$ and $Q$ on a semicircle $\delta$, we say that $P, Q$ and the endpoints of $\delta$ lie counterclockwise if $P, Q$ and one of the endpoints of $\delta$ lie counterclockwise. If a circle touches $\alpha_{m}, \beta_{n}$ and $\gamma$ internally so that the points of tangency of this circle and each of $\beta_{m}, \alpha_{n}$ and $\gamma$ lie counterclockwise, we say that the circle touches $\alpha_{m}, \beta_{n}$ and $\gamma$ appropriately. Also if a semicircle touches $\alpha_{m}$ and $\beta_{n}$, and $\gamma$ at the endpoints so that the points of tangency of the semicircle and each of $\beta_{n}, \alpha_{m}$, and the endpoints lie counterclockwise, then we say that the semicircle touches $\alpha_{m}$, $\beta_{n}$ and $\gamma$ appropriately.

Theorem 2 If $m \neq 0$ and $n \neq 0$, the following three statements are equivalent.

(i) A circle of radius $i_{c}$ touches $\alpha_{m}, \beta_{n}$ and $\gamma$ appropriately. (ii) A semicircle of radius $i_{s}$ touches $\alpha_{m}, \beta_{n}$ and $\gamma$ appropriately.

(iii) $c+2 h=\frac{a+h}{m}+\frac{b+h}{n}$.

Proof. Assume that (i) and $(x, y)$ are the coordinates of the center of the circle in (i). Then we have $(x-$ $m(a+h))^{2}+y^{2}=\left(m(a+h)+i_{c}\right)^{2},(x+n(b+h))^{2}+y^{2}=$ $\left(n(b+h)+i_{c}\right)^{2}$ and $(x-(a-b))^{2}+y^{2}=\left(c-i_{c}\right)^{2}$. Eliminating $x$ and $y$ from the three equations with (1), we get (iii). Conversely we assume (iii), and a circle of radius $i_{c}$ touches $\alpha_{m}, \beta_{n^{\prime}}$ and $\gamma$ appropriately for a real number $n^{\prime}$. Then we have $a+b+2 h=(a+h) / m+(b+h) / n^{\prime}$ just as we have shown, i.e., $n=n^{\prime}$. Hence $\beta_{n}=\beta_{n^{\prime}}$, i.e., (iii) implies (i). Therefore (i) and (iii) are equivalent. The equivalence of (ii) and (iii) is proved similarly.

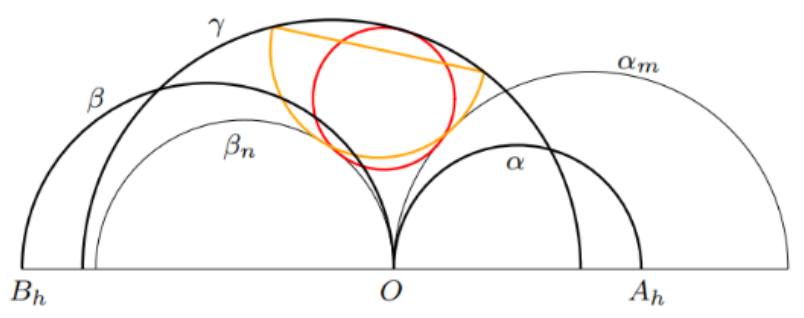

Figure 3: $1<m$ and $0<n<1$.

Theorem 2 does not consider the case in which $\alpha_{m}$ or $\beta_{n}$ coincides with the axis. We consider the case in the next theorem (see Figure 4).

Theorem 3 The following statements hold.

(i) A circle of radius $i_{c}$ touches $\alpha_{m}(m>0)$ externally, $\gamma$ 
internally and the axis if and only if

$m=m_{0}=\frac{a+h}{c+2 h}$

(ii) A semicircle of radius $i_{s}$ touches $\alpha_{m}(m>0)$ and the axis, and $\gamma$ at the endpoints if and only if (3) holds.

(iii) A circle of radius $i_{c}$ touches $\beta_{n}(n>0)$ externally, $\gamma$ internally and the axis if and only if

$n=n_{0}=\frac{b+h}{c+2 h}$.

(iv) A semicircle of radius $i_{s}$ touches $\beta_{n}(n>0)$ and the axis, and $\gamma$ at the endpoints if and only if (4) holds.

Proof. We prove (i). Let $(x, y)$ be the coordinates of the center of the circle of radius $i_{c}$ in (i). Then we have $x=i_{c},(x-m(a+h))^{2}+y^{2}=\left(m(a+h)+i_{c}\right)^{2}$ and $(x-(a-b))^{2}+y^{2}=\left(a+b-i_{c}\right)^{2}$. Eliminating $x$ and $y$ from the three equations with (1), and solving the resulting equation for $m$, we get (3). Conversely, we assume that (3) and a circle of radius $i_{c}$ touches $\alpha_{m^{\prime}}\left(m^{\prime}>0\right)$ externally, $\gamma$ internally and the axis for a real number $m^{\prime}$. Then we have $m^{\prime}=m_{0}=m$ as just we have proved. Therefore $\alpha_{m^{\prime}}=\alpha_{m}$ and the converse is true. The rest of the theorem is proved similarly.

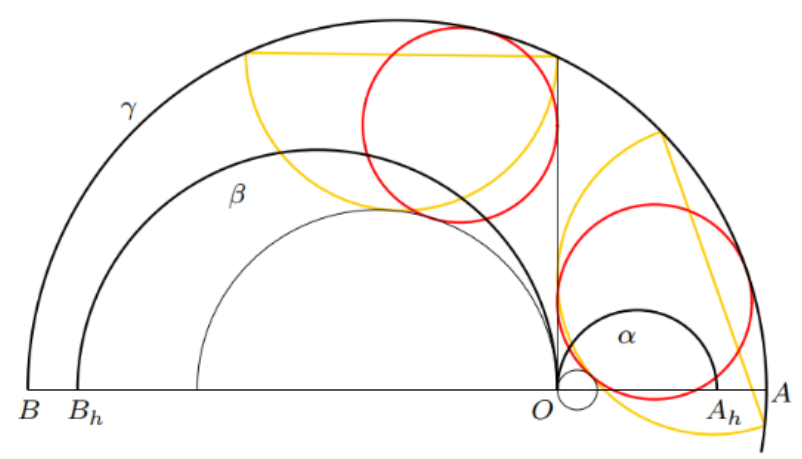

Figure 4

If $m=m_{0}$, then $(a+h) / m=c+2 h$. Therefore if $(b+$ $h) / n_{x}=0$, and $\beta_{n_{x}}$ coincides with the axis, then we can consider that Theorem 2 is true in the case $(m, n)=\left(m_{0}, n_{x}\right)$. Similarly if $n=n_{0}$ and $(a+h) / m_{x}=0$ and $\alpha_{m_{x}}$ coincides with the axis, we can also consider that Theorem 2 holds in the case $(m, n)=\left(m_{x}, n_{0}\right)$. Therefore Theorems 2 and 3 can be unified in this case. We consider about this in section 4 .

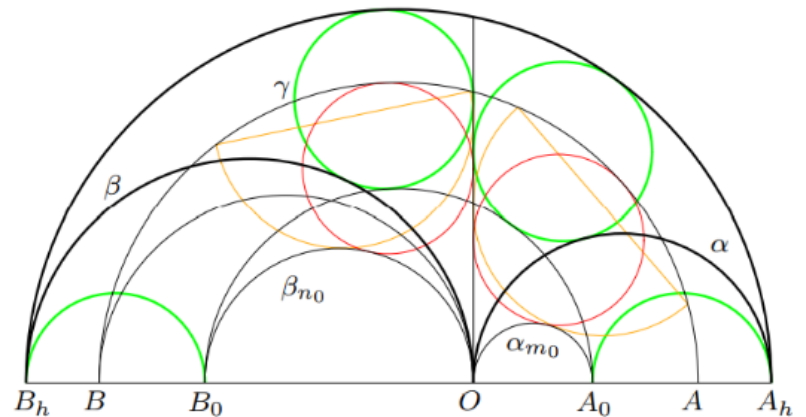

Figure 5

Theorem 4 If $A_{0} O$ and $B_{0} O$ are the diameters of the circles $\alpha_{m_{0}}$ and $\beta_{n_{0}}$, respectively, then the circles of diameters $A_{0} A_{h}$ and $B_{0} B_{h}$ are Archimedean circles of the arbelos made by $\alpha, \beta$ and the semicircle of diameter $A_{h} B_{h}$ constructed on the same side of $A B$ as $\gamma$. Therefore the circle of diameter $A_{0} B_{0}$ is concentric to $\gamma$ and touches the twin circles of Archimedes of the arbelos.

Proof. Since the radius of the circle $\alpha_{m_{0}}$ equals $(a+h) m_{0}=$ $(a+h)^{2} /(c+2 h)$ by (3), the circle of diameter $A_{0} A_{h}$ has radius

$$
(a+h)-\frac{(a+h)^{2}}{c+2 h}=\frac{(a+h)(b+h)}{c+2 h},
$$

which equals the radius of Archimedean circles of the arbelos made by $\alpha, \beta$ and the semicircle of diameter $A_{h} B_{h}$ (see Figure 5). Since the radius of the circle is symmetric in $a$ and $b$, the other circle also has the same radius.

\section{Archimedean semicircles}

In this section we consider another kind of semicircles touching $\gamma$ at the endpoints.

Theorem 5 The semicircle touching $\alpha$ and the axis and $\gamma$ at the endpoints is congruent to the semicircle touching $\beta$ and the axis and $\gamma$ at the endpoints. The common radius equals

$s_{\mathrm{A}}=\frac{1}{2}\left(\sqrt{(c+2 h)^{2}+8 a b}-c-2 h\right)$.

Proof. Let $(s, y)$ be the coordinates of the center of the semicircle touching $\alpha$ and the axis, and $\gamma$ at the endpoints. Then $s$ equals the radius of the semicircle, and we have $(s-(a-b))^{2}+y^{2}+s^{2}=c^{2}$ by Proposition 1 and $(s-(a+h))^{2}+y^{2}=((a+h)+s)^{2}$. Eliminating $y$ from the two equations and solving the resulting equation for $s$, we have $s=s_{\mathrm{A}}$. Since $s$ is symmetric in $a$ and $b$, the other semicircle also has the same radius. 


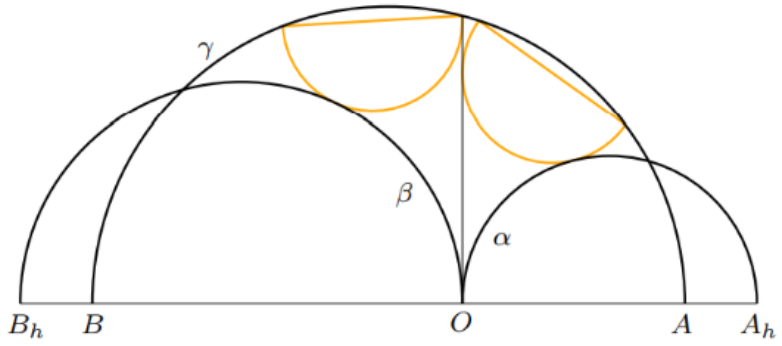

Figure 6

The two congruent semicircles in Theorem 5 may be called the twin semicircles of Archimedes (see Figure 6). A semicircle of radius $s_{\mathrm{A}}$ is called an Archimedean semicircle of $(\alpha, \beta, \gamma)_{h}$ or said to be Archimedean with respect to $(\alpha, \beta, \gamma)_{h}$. Let $w_{k}=\sqrt{a^{2}+k a b+b^{2}}$. Theorem 5 shows that $(\alpha, \beta, \gamma)_{0}$ has Archimedean semicircles of radius $\left(w_{10}-c\right) / 2$.

Theorem 6 Assume that $(m, n) \neq(1,0),(0,1)$ and a semicircle touches $\alpha_{m}, \beta_{n}$ and $\gamma$ appropriately. Then the semicircle is Archimedean with respect to $(\alpha, \beta, \gamma)_{h}$ if and only if

$\frac{1}{m}+\frac{1}{n}=1$.

Proof. Assume that a semicircle of radius $s_{\mathrm{A}}$ touches $\alpha_{m}, \beta_{n}$ and $\gamma$ appropriately and $(x, y)$ are the coordinates of its center. Then we get $(x-m(a+h))^{2}+y^{2}=\left(m(a+h)+s_{\mathrm{A}}\right)^{2}$, $(x+n(b+h))^{2}+y^{2}=\left(n(b+h)+s_{\mathrm{A}}\right)^{2}$, and $(x-(a-b))^{2}+$ $y^{2}+s_{\mathrm{A}}^{2}=c^{2}$. Eliminating $x$ and $y$ from the three equations, we have (6). Conversely we assume (6) and assume that a semicircle of radius $s_{\mathrm{A}}$ touches $\alpha_{m}, \beta_{n^{\prime}}$ and $\gamma$ appropriately. Then we have $1 / m+1 / n^{\prime}=1$. Hence we get $n=n^{\prime}$, i.e., $\beta_{n}=\beta_{n^{\prime}}$. Hence the converse holds.

While we have obtained the next theorem in [4].

Theorem 7 If $(m, n) \neq(1,0),(0,1)$ and a circle touches $\alpha_{m}, \beta_{n}$ and $\gamma$ appropriately, then the circle is Archimedean with respect to $(\alpha, \beta, \gamma)_{h}$ if and only if (6) holds.

By Theorems 6 and 7 we have the next theorem.

Theorem 8 If $(m, n) \neq(1,0),(0,1)$, the following statements are equivalent.

(i) The circle touching $\alpha_{m}, \beta_{n}$, and $\gamma$ appropriately is Archimedean with respect to $(\alpha, \beta, \gamma)_{h}$.

(ii) The semicircle touching $\alpha_{m}, \beta_{n}$, and $\gamma$ appropriately is Archimedean with respect to $(\alpha, \beta, \gamma)_{h}$.

(iii) (6) holds.

It is commonly considered that the circles $\alpha_{0}$ and $\beta_{0}$ are point circles and coincide with the origin $O$. This implies that Theorem 8 is not true in the cases $(m, n)=(1,0),(0,1)$. Therefore Theorems 8 does not consider the case of the twin circles of Archimedes and the case of the twin semicircles of Archimedes. We consider the case in the next section.

\section{Division by zero}

In this section we show that we can consider that the circles $\alpha_{0}$ and $\beta_{0}$ coincide with the axis using recently made definition of division by zero [5].

For a field $F$ we consider the following bijection $\psi: F \rightarrow F$ :

$$
\psi(a)= \begin{cases}a^{-1} & \text { if } a \neq 0 \\ 0 & \text { if } a=0\end{cases}
$$

It is a custom to denote $z \psi(a)$ by $z / a$ if $a \neq 0$, i.e., $z \psi(a)=$ $a / z$ for $a \neq 0$. Following to this, we write

$z \cdot \psi(0)=\frac{z}{0}$ for $\forall z \in F$.

Then we have

$z \cdot \psi(a)=\frac{z}{a}$ for $\forall a, z \in F$.

Especially we have

$\frac{z}{0}=z \cdot 0=0$ for $\forall z \in F$.

Notice that the concept of the reduction to common denominator can not be used for $z / 0$, i.e., we have the following relation in general in the case $b=0$ or $d=0$ :

$$
\frac{a}{b}+\frac{c}{d} \neq \frac{a d+b c}{b d}
$$

We consider the circle $\alpha_{m}$ in the case $m=0$. The circle $\alpha_{m}$ has an equation $(x-m(a+h))^{2}+y^{2}=m^{2}(a+h)^{2}$, or

$-2 m(a+h) x+\left(x^{2}+y^{2}\right)=0$.

This implies $x^{2}+y^{2}=0$ if $m=0$. Hence $\alpha_{0}$ coincides with the origin in this case. On the other hand, 10 can be written as

$-2(a+h) x+\frac{x^{2}+y^{2}}{m}=0$.

Therefore we get $-2(a+h) x=0$, i.e., $x=0$ if $m=0$ by (9), i.e., $\alpha_{0}$ coincides with the axis in this case. Now we can consider that $\alpha_{0}$ is the origin or the axis, or the axis as the union of them. Similarly $\beta_{0}$ can be considered as the origin or the axis.

We can now consider that $\alpha_{0}$ and $\beta_{0}$ coincide with the axis. Then Theorem 2 holds in the case $(m, n)=\left(m_{0}, 0\right),\left(0, n_{0}\right)$ by (9). Also Theorem 8 holds in the case $(m, n)=$ 
$(1,0),(0,1)$. Our current mathematics avoids to consider (9). But our above observation shows that (9) is useful.

Division by zero was founded by Saburou Saitoh in 2014. He has been making a list of successful example applying division by zero and its generalization called division by zero calculus, and there are more than 1200 evidences. It shows that a new world of mathematics can be opened if we admit them. For an extensive reference of division by zero and division by zero calculus including those evidences, see [5].

\section{Aida arbelos}

Aida (1747-1817) considered a figure consisting of two touching semicircles at their midpoints and the circle passing through the endpoints of the semicircles [1] (see Figure 7). He gave several notable properties of this figure, which are summarized in [3]. We conclude this paper by considering special circles and special semicircles for this figure.

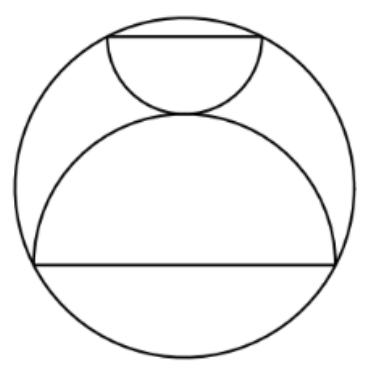

Figure 7: Aida's figure.

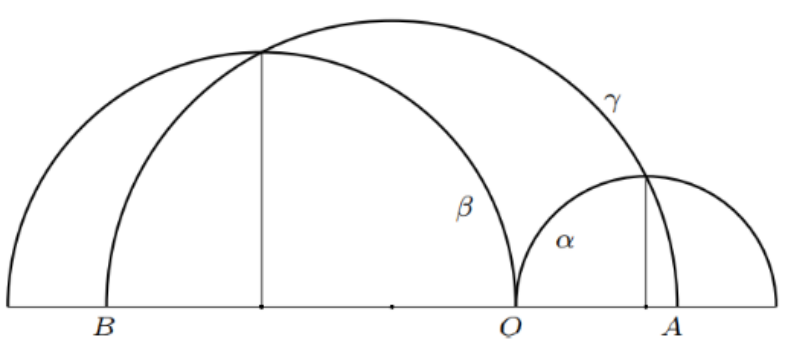

Figure 8: Aida arbelos.

Aida's figure is obtained from $(\alpha, \beta, \gamma)_{h}$, when $h=r_{\mathrm{A}}$ [3], or

$h=\frac{a b}{c+h}$.

Because (12) is equivalent to

$r_{\mathrm{A}}=h=\frac{1}{2}\left(w_{6}-c\right)$, and (13) implies that the farthest points on $\alpha$ and $\beta$ from $A B$ lie on $\gamma$, where recall $w_{k}=\sqrt{a^{2}+k a b+b^{2}}$. In this case we call $(\alpha, \beta, \gamma)_{h}$ an Aida arbelos (see Figure 8). Replacing $h$ in the denominator of the right side of $(12)$ by the right side of (12) repeatedly, we get a continued fraction expansion of $r_{\mathrm{A}}$ for the Aida arbelos:

$$
r_{\mathrm{A}}=\frac{a b}{c+h}=\frac{a b}{c+\frac{a b}{c+h}}=\frac{a b}{c+\frac{a b}{c+\frac{a b}{c+\ddots}}} .
$$

We assume $h \geq 0$. Let $\bar{\alpha}$ and $\bar{\beta}$ be the semicircles of diameters $A O$ and $B O$, respectively, constructed on the same side of $A B$ as $\gamma$, i.e., $\bar{\alpha}, \bar{\beta}$ and $\gamma$ form $(\alpha, \beta, \gamma)_{0}$. The incircle of the curvilinear triangle made by $\alpha, \bar{\alpha}($ resp. $\beta, \bar{\beta})$ and the radical axis of $\alpha$ (resp. $\beta)$ and $\gamma$ has radius $\left(1 / r_{\mathrm{A}}+1 / h\right)^{-1}$ for $(\alpha, \beta, \gamma)_{h}$ [4]. Therefore the radius equals $r_{\mathrm{A}} / 2$ for the Aida arbelos. The circles are denoted by green in Figure 9. The circle touching $\alpha$ or $\beta$ externally, $\gamma$ externally and the axis has radius $a b / h$ for $(\alpha, \beta, \gamma)_{h}$ [4]. Hence the radius equals $a b / r_{\mathrm{A}}=c+r_{\mathrm{A}}$ for the Aida arbelos by (12). The circles are denoted by magenta in Figure 9.

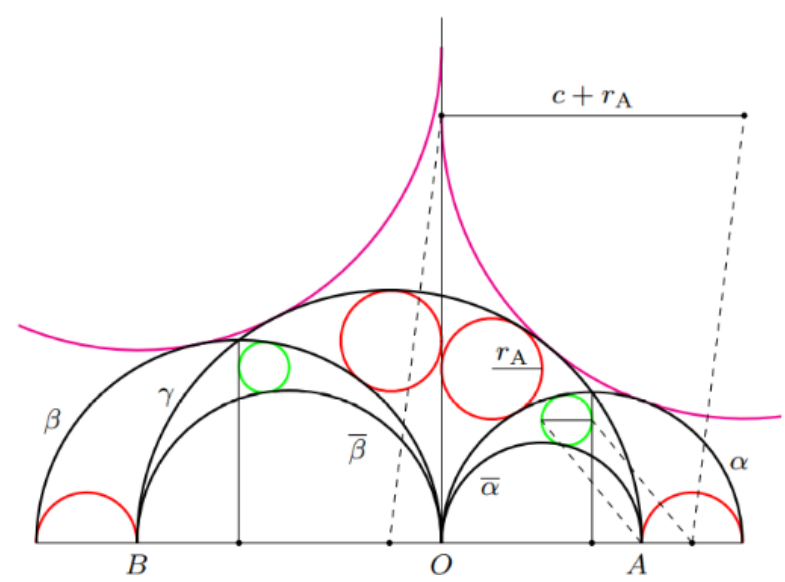

Figure 9: The green circles have radius $r_{\mathrm{A}} / 2$.

Substituting (13) in (5), we get that the radius of Archimedean semicircles of the Aida arbelos equals

$$
s_{\mathrm{A}}=\frac{1}{2}\left(w_{14}-w_{6}\right) \text {. }
$$

Since $i_{c}=w_{6} h / c$ for the Aida arbelos [3], we get that the inradius of the Aida arbelos equals

$$
i_{c}=\frac{w_{6}\left(w_{6}-c\right)}{2 c}
$$

by 13 . Therefore we have

$$
i_{c}+r_{\mathrm{A}}=\frac{2 a b}{c} \text {. }
$$


Hence the sum of $i_{c}$ and $r_{\mathrm{A}}$ for the Aida arbelos equals the diameter of the Archimedean circle of $(\alpha, \beta, \gamma)_{0}$. Let $u=\left(w_{6}^{4}+16 a^{2} b^{2}\right)^{1 / 4}$.

Theorem 9 If the insemicircle of the Aida arbelos has center of coordinates $\left(x_{s}, y_{s}\right)$, we have

$i_{s}=\frac{u^{2}-c^{2}}{2 w_{6}}$,

$\left(x_{s}, y_{s}\right)=\left(\frac{(b-a) i_{s}}{w_{6}}, \frac{4 a b \sqrt{4 a b+u^{2}}}{w_{6}^{2}}\right)$.

Proof. By (2) and (13), we get (14). Solving the equations $\left(x_{s}-(a+h)\right)^{2}+y_{s}^{2}=\left((a+h)+i_{s}\right)^{2}$ and $\left(x_{s}+(b+h)\right)^{2}+$ $y_{s}^{2}=\left((b+h)+i_{s}\right)^{2}$ with (14), we get 15).

The next theorem shows that the result for the insemicircle of $(\alpha, \beta, \gamma)_{0}$ obtained in [2] also holds for the Aida arbelos (see Figure 10).

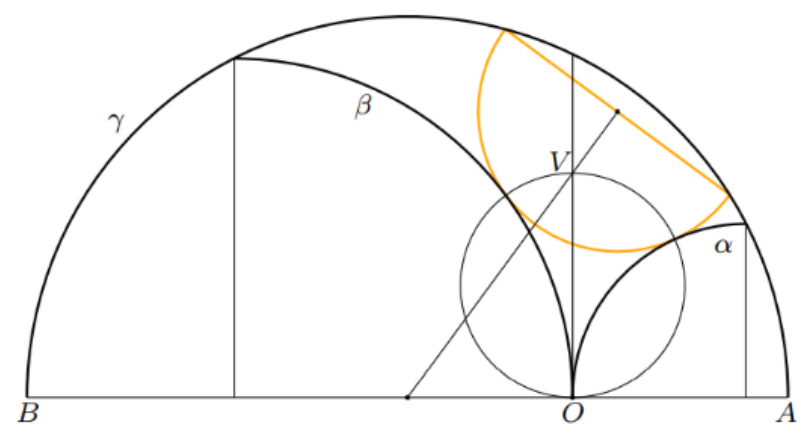

Figure 10

Theorem 10 If the line joining the centers of $\gamma$ and the insemicircle of the Aida arbelos meets the axis in a point
$V$, then the circle of diameter $O V$ is orthogonal to the insemicircle. Hence the circle passes through the points of tangency of two of $\alpha, \beta$ and the insemicircle.

Proof. From (13) and (15), the circle of diameter $O V$ has radius

$$
r_{v}=\frac{4 a b \sqrt{4 a b+u^{2}}}{w_{10}^{2}+u^{2}}
$$

and the center of coordinates $\left(0, y_{v}\right)=\left(0, r_{v}\right)$. Then we have $\left(x_{s}-0\right)^{2}+\left(y_{s}-y_{v}\right)^{2}=r_{v}^{2}+i_{s}^{2}$.

\section{References}

[1] Aida ed., Sampō Chikusaku Jutsu, Volume 2, Tohoku University Digital Collection.

[2] H. OKumuRA, The arbelos in Wasan geometry, the inscribed semicircle in the arbelos, Sangaku J. Math. 5 (2021), 1-8.

[3] H. OKumura, Wasan geometry. In: B. Sriraman ed., Handbook of the Mathematics of the Arts and Sciences, Springer, 2020, 1-52, doi: 10.1007/978-3-31970658-0_122-1

[4] H. OKumuRA, The arbelos with overhang, $K o G \mathbf{1 8}$ (2014), 19-27.

[5] S. SAITOH, Introduction to the division by zero calculus, Scientific Research Publishing, 2021.

\section{Hiroshi Okumura}

orcid.org/0000-0003-4332-0189

e-mail: hokmr@yandex.com

Takahanadai Maebashi Gunma 371-0123, Japan 